\title{
FACE RECOGNITION TECHNIQUE BASED ON MODULAR ICA APPROACH
}

\author{
CAO Wen-ming ${ }^{1}$ LU Fei $^{1}$ YUAN Yuan' ${ }^{1}$ WANG shuojue ${ }^{2}$ \\ 'Institute of Intelligent College of Information Engineering, Zhejiang University of \\ Technology, Hangzhou 310014, China, ${ }^{2}$ Institute of Semiconductors, Chinese Academy of \\ Science, Beijing, 100083,China
}

\begin{abstract}
In this paper, a face recognition algorithm based on modular ICA approach is presented. Compared whit conventional ICA algorithm, the proposed algorithm has an improved recognition rate for face images with large variations in lighting direction and facial expression. In the proposed technique, the face images are divided into smaller sub-images and the ICA approach is applied to each of these sub-images. Since some of the local facial features of an individual do not vary even when the pose, lighting direction and facial expression vary, we expect the proposed method to be able to cope with these variations. The accuracy of the conventional ICA method and modular ICA method are evaluated under the conditions of varying expression, illumination and pose using Yale face database[1].
\end{abstract}

Keywords: ICA; Face recognition: Modular ICA

\section{INTRODUCTION}

Face recognition is a difficult problem because of the generally similar shape of faces combined with the numerous variations between images of the same face. The image of a face changes with facial expression, age, viewpoint, illumination conditions, noise etc. The task of a face recognition 
system is to recognize a face in a manner that is as independent as possible of these image variations.

In this paper, a face recognition algorithm based on modular ICA approach is presented. Compared whit conventional ICA algorithm, the proposed algorithm has an improved recognition rate for face images with large variations in lighting direction and facial expression.

\section{REVIEW OF THE ICA METHOD}

\subsection{The principle of ICA}

The ICA is usually used to process statistic signal. The principle of this algorithm is to denote a set of stochastic variables by a set of basis functions which are independent of each other. Let the observation signals from $M$ channels are $x_{l}(i=1,2, \cdots, M)$, and each signal is composed of $N$ independent signal $s_{j}(j=1,2, \cdots, N)$, namely:

$x_{i}=a_{i 1} s_{1}+a_{i 2} s_{2}+\cdots+a_{i N} s_{N} \quad(i=1,2, \cdots, M)$. Which can be represented as follow formula:

$\mathrm{X}=A \bullet S, X=\left(x_{1}, x_{2}, \cdots, x_{M}\right), S=\left(s_{1}, s_{2}, \cdots, s_{N}\right)$,

$A$ is an unknown mixed matrix, $S$ is an independent signal. After we figure out the mixed $A$, we can get $S=A^{-1} \bullet X=W \bullet X . \mathrm{W}=A^{-1}$ was defined as separate matrix. There are two methods to realize the ICA algorithm: 1) Minimize or maximize assessment function, which need complex vector computation;2) Self-adaptive algorithm based on random grads lowering, which converges very slowly. Hyvärinen et al [2][3][4][5] putted forward a" Fast and Robust Fixed-Point Algorithms for Independent Component Analysis", which are very efficient.

According to [2], every independent heft can be recognized as an extremum of a peak. The peak of the projection of the stochastic variables $X$ on a vector can be represented as follow:

$$
\operatorname{Kurt}\left(w^{T} X\right)=E\left\{y^{T} X\right\}^{4}-3\left(E\left\{w^{T} X\right\}^{2}\right)^{2}
$$

The mean of $X$ is zero, and $E\left\{\left(w^{T} X\right)^{2}\right\}=1$. We can get iteration formula:

$$
\begin{gathered}
w^{*}(k)=C^{-1} E\left\{X\left[w(k-1)^{T} X\right]^{3}\right\}-3 w(k-1) \\
w(k)=w^{*}(k) /\left[w^{*}(k)^{T} C w^{*}(k)\right]^{1 / 2}
\end{gathered}
$$

$C=E\left\{X X^{T}\right\}$ is a covariance matrix; $k$ is the number of iterations. If there is a stable extremum at peak $\operatorname{Kurt}\left(w^{T} X\right)$, then the independent heft of stochastic variables $X$ is: 


$$
s_{i}= \pm w_{i}^{T} X \quad(i=1,2, \cdots, N)
$$

\subsection{Face recognition based on ICA}

Let $x_{i}$ represents the i-th image of a face. There are $n$ stochastic variable in training set, namely $x_{i}(i=1,2, \cdots, n)$. Each $x_{i}$ is composed of $m$ unknown independent heft. Then we can get $X=A \bullet S$, $x_{i}=\left[a_{i 1}, a_{i 2}, \cdots, a_{i m}\right] \bullet\left[s_{1}, s_{2}, \cdots, s_{m}\right]^{\prime}$.The independent heft $s_{j}(j=1,2, \cdots, m)$ describes the feature of a face.

When we test the face vector test, at first, we get the weight vector by projection of test on $S$ :

$$
V=S \bullet \text { test }
$$

Then we compute Euclid norm:

$$
\begin{aligned}
& d_{i}=\left\|V-W_{i}\right\|, i=1,2, \ldots N \\
& W_{i}=S \bullet x_{i} \quad S=\left[s_{1}, s_{2}, \cdots s_{m}\right]^{T}, \quad i=1,2, \ldots N
\end{aligned}
$$
test

The i-th class corresponding to the least ${ }_{i}$ is the same class with face

\section{FACE RECOGNITION BASED ON MODULAR ICA}

\subsection{Modular ICA}

The ICA based face recognition method is not very effective under the conditions of varying pose and illumination, since it considers the global information of each face image and represents them with a set of weights. Under these conditions the weight vectors will vary considerably from the weight vectors of the images with normal pose and illumination, hence it is difficult to identify them correctly. On the other hand if the face images were divided into smaller regions and the weight vectors are computed for each of these regions, then the weights will be more representative of the local information of the face. When there is a variation in the pose or illumination, only some of the face regions will vary and the rest of the regions will remain the same as the face regions of a normal image. Hence weights of the face regions not affected by varying pose and illumination will closely match with the weights of the same individual's face regions under normal conditions. Therefore it is expected that improved recognition rates can be obtained by following the modular ICA approach. We expect that if the face images are divided into very small region the global information of the face may be lost and the accuracy of this method may deteriorate. 
There are $N$ face images in our face database. Let vector $x_{i}(i=1,2, \ldots N)$ represent the face images in our face database. In this method, each image in the training set is divided into $H$ smaller images (as figure 1). Hence it can be represented as the follow formula:
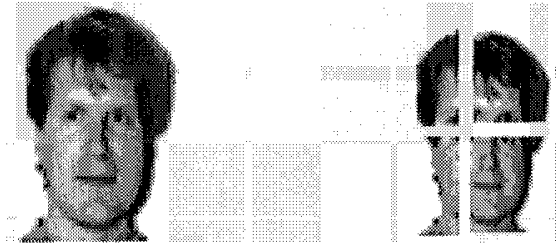

Figure 1. Separate an image into four sub-images.

$$
\begin{aligned}
& x_{i j}(m, n)=x_{i}\left(\frac{W}{\sqrt{H}}(j-1)+m, \frac{W}{\sqrt{H}}(j-1)+n\right) \\
& i=1,2, \cdots N j=1,2, \cdots H, m=1,2, \cdots L / \sqrt{N}, n=1,2, \cdots L / \sqrt{N}
\end{aligned}
$$

Then, we can get independent hefts by the ICA method.

\subsection{Classification}

Let the independent heftses derived from sub-images are $S_{i}(j=1,2, \cdots H)$. When we test the face vector test, at first, we separate it into $H$ sub-images. Then, we compute the projection weight vectors of test ${ }_{j}$ on $S_{j}(j=1,2, \cdots H)$ :

$$
V_{j}=S_{j} \bullet \text { test }_{j} \quad j=1,2, \cdots, H
$$

And then, compute the mean Euclid norm:

$$
\begin{gathered}
d_{i}=\frac{1}{H} \sum_{j=1}^{H} d_{i j} \\
d_{i j}=\left\|V_{j}-W_{i j}\right\| \quad i=1,2, \ldots N, \quad j=1,2, \cdots, H \\
W_{i j}=S_{j} \bullet x_{i j} \quad i=1,2, \ldots N, \quad j=1,2, \cdots, H
\end{gathered}
$$
test

The $i$-th class corresponding to the least $d_{i}^{\prime}$ is the same class with face

\section{YALE FACE DATABASE [1]}

The Yale database has 165 images of 15 adults, 11 images per person. The face images vary with respect to facial expression and illumination. The 
images have normal, sad, happy, sleepy, surprised, and winking expressions. There are also images where the position of the light source is at the center, left and right. In addition to these, there are images with and without glasses. Out of the 11images of a person, only eight were used for training and the remaining three were used to test the recognition rates.Fi g.2 and 3 show the set of images of a person used for training and testing respectively. The choice of the training and test images was made to facilitate comparison of performance of both the methods for test images with uneven illumination and partial occlusion.

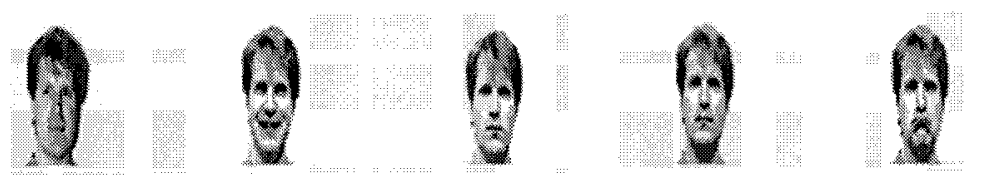

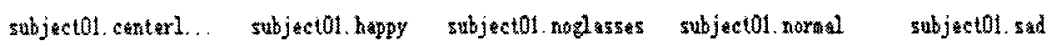

Figure 2. Images for training
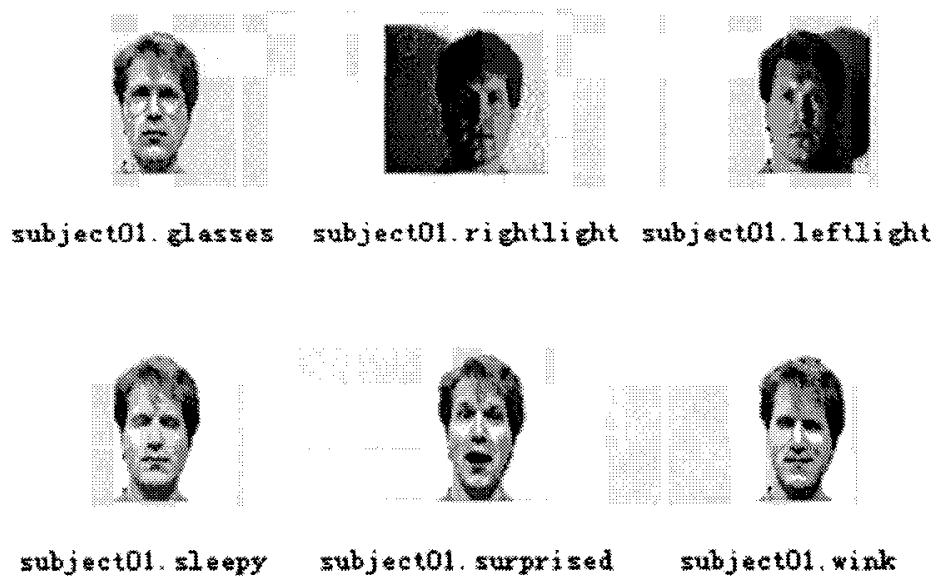

Figure 3. Images for testing

In our experiment, each image is stretched to $64 \times 64$ pixels, and then normalized as follow:

$$
x_{i}{ }^{\prime}=\frac{s \operatorname{var}}{\operatorname{var}}\left(x_{i}-\text { mean }\right)+\text { smean }
$$

svar is the expecting mean square error, var is the square error of this image, smean is the expecting mean, and mean is the mean of this image. 


\section{EXPERIMENT RESULT}

\begin{tabular}{|c|c|c|c|}
\hline $\begin{array}{l}\text { Recognition } \\
\text { method }\end{array}$ & $\begin{array}{l}\text { Face recognition } \\
\text { based on ICA }\end{array}$ & $\begin{array}{l}\text { Face recognitio } \\
\text { modular ICA }\end{array}$ & pased on \\
\hline \multirow{4}{*}{$\begin{array}{l}\text { Recognition } \\
\text { Rate }\end{array}$} & \multirow[t]{4}{*}{$81.11 \%$} & $\begin{array}{l}\text { Separate an } \\
\text { image into } 2 \text { sub- } \\
\text { images(separate by } \\
\text { up and down) }\end{array}$ & $80.00 \%$. \\
\hline & & $\begin{array}{l}\text { Separate an } \\
\text { image into } 2 \text { sub- } \\
\text { images(separate by } \\
\text { left and right) }\end{array}$ & $95.56 \%$ \\
\hline & & $\begin{array}{l}\text { Separate } \\
\text { image into } 4 \text { sub- } \\
\text { images }\end{array}$ & $94.44 \%$ \\
\hline & & $\begin{array}{l}\text { Separate an } \\
\text { image into } 16 \text { sub- } \\
\text { images }\end{array}$ & $93.33 \%$ \\
\hline
\end{tabular}

A typical example:

With the ICA method, fig 4 (a) was classified to 4 (b). But with the modular ICA, fig 4 (a) was classified to 4 (c).

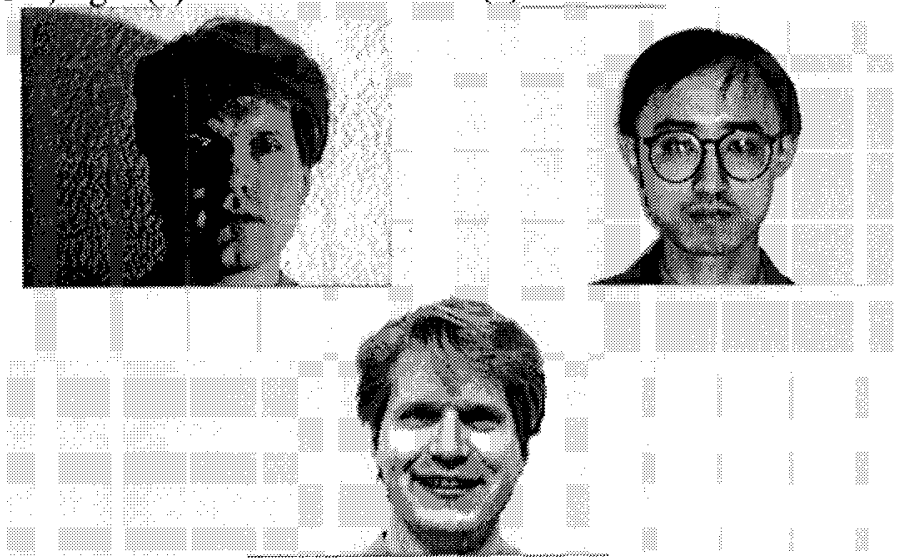

Figure 4. (a) subject01.rightlight (b) subject08.centerlight (c) subject01.happy

\section{CONCLUSION}

We can conclude from the data that: 
(1) . Because the position of the light source vary horizontally not vertically, the recognition rate of modular ICA is less than ICA when images are separated by up and down; the recognition rate of modular ICA is highest when images are separated by up and down. This phenomenon denotes that modular ICA keeps a high recognition rate when the face images vary with respect to illumination.

(2). When the images are separated into 16 sub-images, the recognition rate is lower than when the images are separated into 4 sub-images. That is because there is little global information when a image is separated into 16 sub-images.

To sum up, face recognition based on modular ICA is much better than based on ICA, especially for face images with large variations in lighting direction and facial expression.

\section{REFERENCE}

1. Yale face database, $<$ http://cvc.yale.edu/projects/yalefaces/yalefaces.html $>$

2. A. Hyvärinen. Fast and Robust Fixed-Point Algorithms for Independent Component Analysis. IEEE Transactions on Neural Networks 10(3):626-634, 1999.

3. A. Hyvärinen and E. Oja. A Fast Fixed-Point Algorithm for Independent Component Analysis. Neural Computation, 9(7):1483-1492, 1997.

4. A. Hyvärinen and E. Oja. Independent Component Analysis: Algorithms and Applications. Neural Networks, 13(4-5):411-430, 2000.

5. A. Hyvarinen . Independent component analysis : A tutorial [OL ]. http ://www. cis. hut . fi/ projects/ ica/ , IJCNN99-tutori-al2.html, 2002. 